\title{
The System of Controlling the Robot by the Smart Phones based on the Bluetooth
}

\author{
Zhenghua Xin ${ }^{1}$, Guolong Chen ${ }^{1}$, Qixiang Song ${ }^{1}$, Liangyi $\mathrm{Hu}^{2}$, Hongmei Lu ${ }^{1}$, Pei \\ Jiang $^{1}$, Yayun Sun ${ }^{1}$ and Lei Chen ${ }^{1}$ \\ ${ }^{1}$ School of Information and Engineering, Suzhou University, Anhui Province, China \\ ${ }^{2}$ School of Economics and Management, Suzhou University, Anhui Province, China \\ ${ }^{1} 18955711392$ \\ 1begin0000@qq.com \\ Abstract
}

\begin{abstract}
In recent years, more and more elderly are living lonely. The project has achieved for them or children to control the robot in the intelligent phones by the Bluetooth serial. It has finished the software about commanding the robot in the intelligent phones based on the android frame. The ultimate function is to realize the smart phone to control the robot via Bluetooth to do a series of actions, such as walking forward and backward, turning left and right, playing soccer, squatting and standing up and according to the corresponding rhythm. The project realizes the handheld control des gn of controlling applications based on Android system. Its core is setting the serial mode of Bluetooth on the Android terminal, and sending the instructions. This will greatly facilitateapplications of the robot in the field of the smart
\end{abstract} home.

Keywords: Bluetooth; the robot, the intelligent phones

\section{Introduction}

The intelligent autonomous mobile robot has the ability to avoid obstacle, detect the motion and perceive the skin. It can do memory computing, the facial recognition, the sound detection, positioning and network communications. Robots can be used as a small family butler in the intelligent home system. The user can give commands directly to the robot to control home electric equpment through the intelligent phone. Robot moves according to the instructions. So the user can remotely control the robot to visit every corner of the home, instead of the fixed cameras in every room. The paper [1] designs a fuzzy logic system and proposes an obstacle avoidance algorithm for a path planning in unknown environment for a mobile robot. The ultrasonic sensors are employed for detecting the distance to obstacles and their positions. An angular velocity control for left and right wheels is implemented by a fuzzy og system. Another new rule table that is induced from the consideration of the distance to obstacles and the angle between the robot and the goal is proposed. Others propose to use robots to replace dentists for completing orthodontic wires bending. Quantitative model of orthodontic wires with canine eminence is established based on Power function model [2].

The short-range wireless communication is suitable for indoor communications. The Bluetooth, the infrared and the wireless sensor net communication is popular [3-6]. The smart phones have embedded the Bluetooth based on the android frame [7]. Through the Androidbased smart phone, the livestock temperature information through images collected from thermal image camera to diagnose in advance detects the livestock disease status [8]. The 
Bluetooth can be applied in this intelligent home system. Of course, the wireless communication based on the Zigbee protocol can get the information from sensors which the robot has. The set of modeled contexts proposed by the paper [9] includes home domainbased contexts and social relationship-based contexts. Using ontology-based context awareness model can provide situation and social-aware health care services in much pervasive way. The chances of surviving a fall, heart attack or stroke are much greater if the senior gets help within an hour. The project designed and developed a smart elderly home monitoring system (SEHMS) [10]. An Android-based smart phone with 3-axial accelerometer is used as the remote medical device which could detect a fall of the carrier. The smart phone is then connected to the monitoring system via WiFi. A graphical user interface (GUI) is developed as the monitoring system which exhibits the information. In addition, this project implemented a remote panic button by using the same android based smart phone.

The elderly and children can stay independently and joyfully in their own horne with the interaction with the robot by their handheld smart phone.

\section{The Hardware of the System}

The equipment of the project uses the serial of the MINIRobot ROBONOVA II robots. The RB Bluetooth Transceiver Bluetooth module is important frot the Robobase Company. The robot is equipped with a model of the MR-C3024EX MCU. This model has a definable transmission microcontroller RS232 interface to extend the functionality. The RB Bluetooth transceiver also uses the RS232 interface. It supports for the Bluetooth 2.1 specification through the serial devices. The default transmission rate is $115200 \mathrm{bps}$. The system of Robots uses the ROBOBASIC programming language from MINIRobot. The ROBOBASIC language is more concise, simpler and easier to use than the $\mathrm{C}$ language which is also used as robot programming language.

To finish the system, it is essential to build the robot development environment. First, the robot programming software of the roboBASIC MF v2.80 needs to be installed. Second, it is necessary to install the robot serial driver, the Windows Driver of the USB 2.0 TO RS232 and the PC Driver.

Since the system of the robot is controlled by the infrared remotely. After the analysis of MR-C3024FX microcontroller circuit, the processing unit of the infrared receiver in the robot system utilizes the ETX pin osend data to the ERX pin of the MR-C3024FX SCM. The ERX is the pin of receiving RS232 signals in the SCM of the Figure 1.

In the Figure 1, the pins from PORT0 to PORT4 and from PORT6 to PORT8 are connecting with the eight servos which are fixed in the left body of the robot to move. To control the right body of the robot to move well, the pins from PORT12 to PORT14 and from PORT18 toPORT22 need to be linked correctly. The pin PORT15 is to control the light of the back. The pin PORT16 is to control the servo in the head. The webcam is the eye of the robot. It is controlled by the pin ETX, ERX and PORT10. And its received images can be sayed in the personal computer. The pin AD2 and AD3 is the receiver. Attaching the AD4, $\mathrm{AD} 5, \mathrm{AD} 0$ and $\mathrm{AD} 1$ with the sensors in the right body of the robot correctly is very important. The AD7, PORT10, PWM0 and LCD control the devices in the front of the robot. The PORT11 is linking with the servo in the neck. 


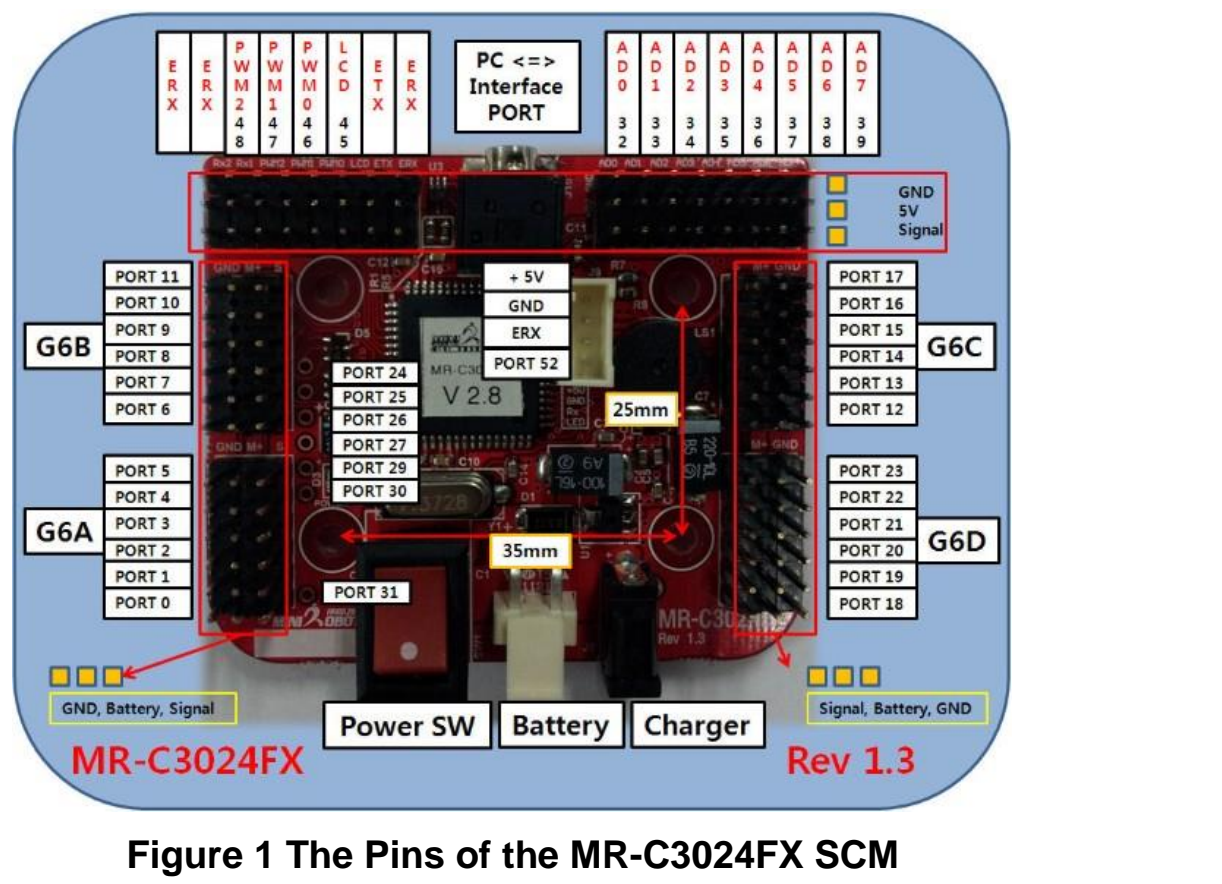

The project team got the value of the received signal processing unit from the transmission between the infrared controller and MR-C3024FX SCM. It has been done by the converter controller from the USB to the RS232TTLyia the computer. After the analysis of the control code, the default transmission mode of the robot is as follows. The baud rate is $4800 \mathrm{bps}$. The data bats are 8Bit data. There is no parily and 1 Stop bit. The ETX pin in the infrared receiver processing unit is connected to the RX pin in the USB to RS232TTL controller. And other test circuits are connected correctly. Then the data are obtained through the serial debugging assistant. After the analysis of the received data, the ASCII value of the infrared remote control key is confirmed. So the MR C3024FX microcontroller is transmitted in ASCII to complete the communication.

After obtaining the communication and transport rules of the MR-C3024FX microcontroler, the ERX pireceives and processes the information. It is directly connected to the TX pin which is part of the computer's USB to RS232TTL controller. The ASCII of a key is sent to the MR- $3024 \mathrm{FX}$ microcontroller through the serial port debugging assistant. But the robot will notact.

There is an EXX pin on the single-chip MR-C3024FX. The pin is an output port of the RS232 interface on the MR-C3024FX microcontroller and ETX. Then the ERX and ETX pin is connecting to the TX and the RX pin of the USB controller on RS232TTL respectively. The corresponding ASCII of the key is sent by the serial debugging assistant. The robot performs the corresponding action. The MR-C3024FX microcontroller communicates with the inf ared receiver processing unit. It is a two-way communication.

So the MR-C3024FX microcontroller completes the communication through the ETX and ERX pin. The ETX pin is responsible for the output and ERX is responsible for input. Then the program for the robot is written as follows.

\section{DIM A AS BYTE}

MAIN: 
ERX 115200, A, MAIN 'The baud rate of the ERX will be set $115200 \mathrm{bps}$ pin. If the value is received, then it will be deposited to the A variable. Otherwise the program will go to the MAIN function to proceed.

ETX 115200, A 'The baud rate of the ETX pin is set to $115200 \mathrm{bps}$. The value of A variable is sent out through the ETX pin.

GOTO MAIN 'It means to go to MAIN to continue.

The instruction in the form of ASCII is sent to the MR-C3024FX microcontroller by the serial debugging assistant. It will be displayed in the dialog of the serial debugging assistant. It is successful to send data to the MR-C3024FX through the RS232.

The default baud rate is $115200 \mathrm{bps}$ for the RB Bluetooth Transceiver Bluetooth module. The four pins of $5 \mathrm{~V}$, GND, RXD, TXD in the RB Bluetooth Transceiver Bluetooth module are connected to four pins of $5 \mathrm{~V}, \mathrm{GND}, \mathrm{TX}, \mathrm{RX}$ in the USB to RS232TTL controller. The Bluetooth transceiver is connected by the USB with RB Bluetooth Transceiver Bluetooth module. Then two serial debugging assistants are opened. One is connected with the Bluetooth controller and the other is connected with the port of the RB Bluetooth Transceiver Bluetooth module. So the data is sen from a serial port debugging assistant. The other serial debugging assistant can recerve the data successfully.

After the test of RB Bluetooth Transceiver Bluetooth module, the RB Bluetooth Transceiver Bluetooth module is connected to the MR-C3024EX microcontroller. The pin is shown as the following Table 1.

\section{Table 1. The connections between the MR-C3024FX Microcontroller and the} Bluetooth

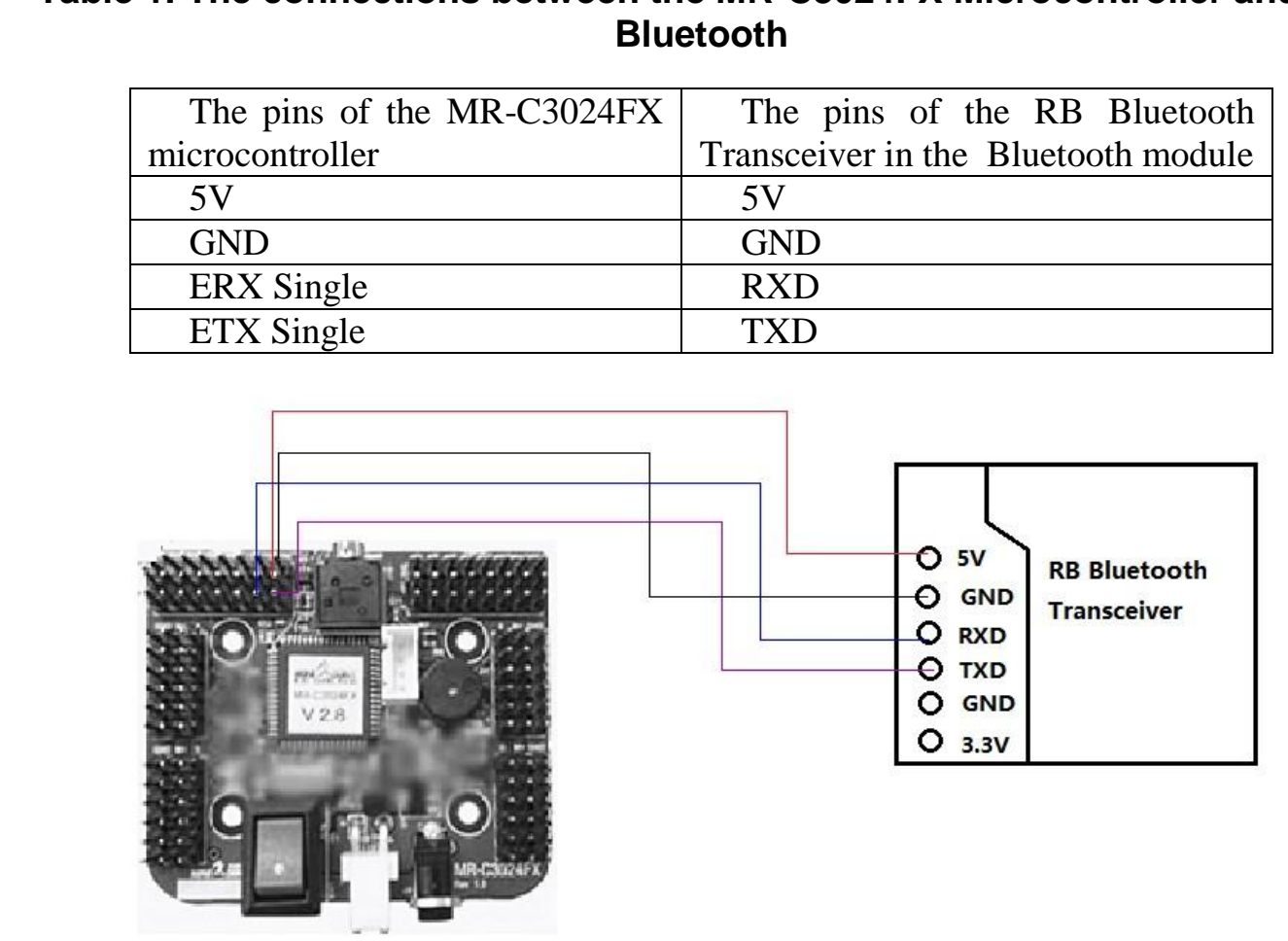

Figure 2. Physical Map of Connecting 
In the Figure 2, the detailed pins in the MR-C3024FX micro-controller and the RB Bluetooth Transceiver are present.

The DuPont lines in four colors connect between the pins 5V, GND, RXD and TXD in the RB Bluetooth Transceiver and the MR-C3024FX micro-controller in the Figure 3. Other pins are connecting with the webcam. Then the operator can control the robot to inspect everything in each room with the webcam by the smart phone.
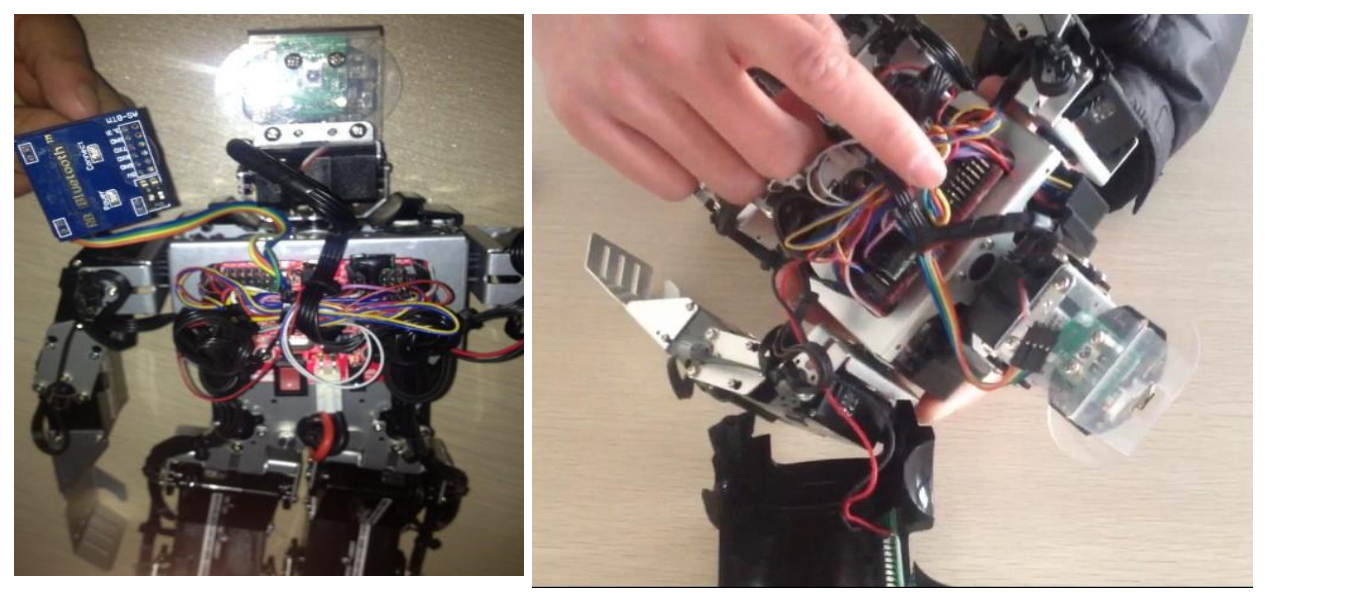

Figure 3. The Robot System after the Connections

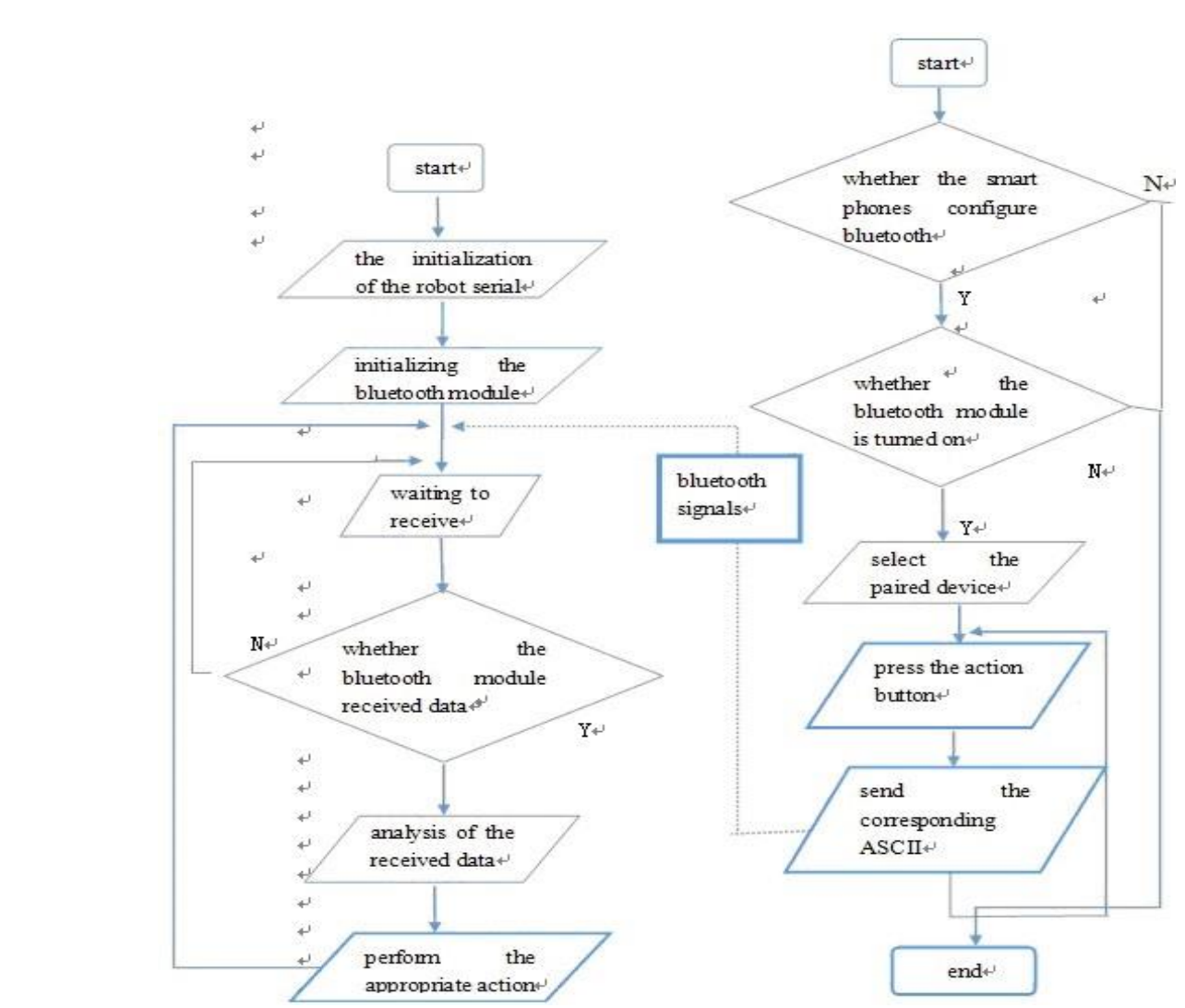

Figure 4. The Flow Chart of the Program 
The robot program pairs the Bluetooth transceiver and RB Bluetooth Transceiver Module. In the serial debugging assistant, the data is sent to the RB Bluetooth Transceiver Bluetooth module. And the transceiver successfully receives the data. The program which can control the robot to act remotely is downloaded. The ASCII via its serial debugging assistant is sent to the RB Bluetooth Transceiver Bluetooth module. The robot successfully performs the corresponding action.

The Figure 4 gives the system flow chart of the robot and the intelligent phone. The left of the Figure 4 is the connection procedure with the Bluetooth device. The right is the running step of the android phone system.

\section{The Software and Results}

The software is based on the android development environment. To build the development environment, the Java jdk, the related software is configured to form the basic environment. Then the android SDK is installed. GOOGLE has integrated the latest version of the SDK and Eclipse Android Developer Tools (ADT). In addition, creating AVD can test the virtual devices of the Android applications.

The initial interface on the smart phone after the running the software is shown as in the Figure 5. The user first presses the searching Bluetooth button on the telephone to find the Bluetooth device. Then she/he clicks the connecting robot, and the status of not connected will be changed. The operator can command the robot to walkforward, to walk backward, to turn left, to turn right and to play football and so on shown as in the Figure 6. In the Figure 7, the robot is controlled to crouch to look for the tabletennis. In the indoor environment, football is too large to suit to be played The robotean inchne to adjust itself to hit the target iteratively. The operator can catch the robot's kicked ball. The elderly control the robot by operating the smart telephone to walk forwardor crouch to get something which is out of their control. When they are lonely, they can play table tennis with the robot. In the interaction with the robot, they are happy.

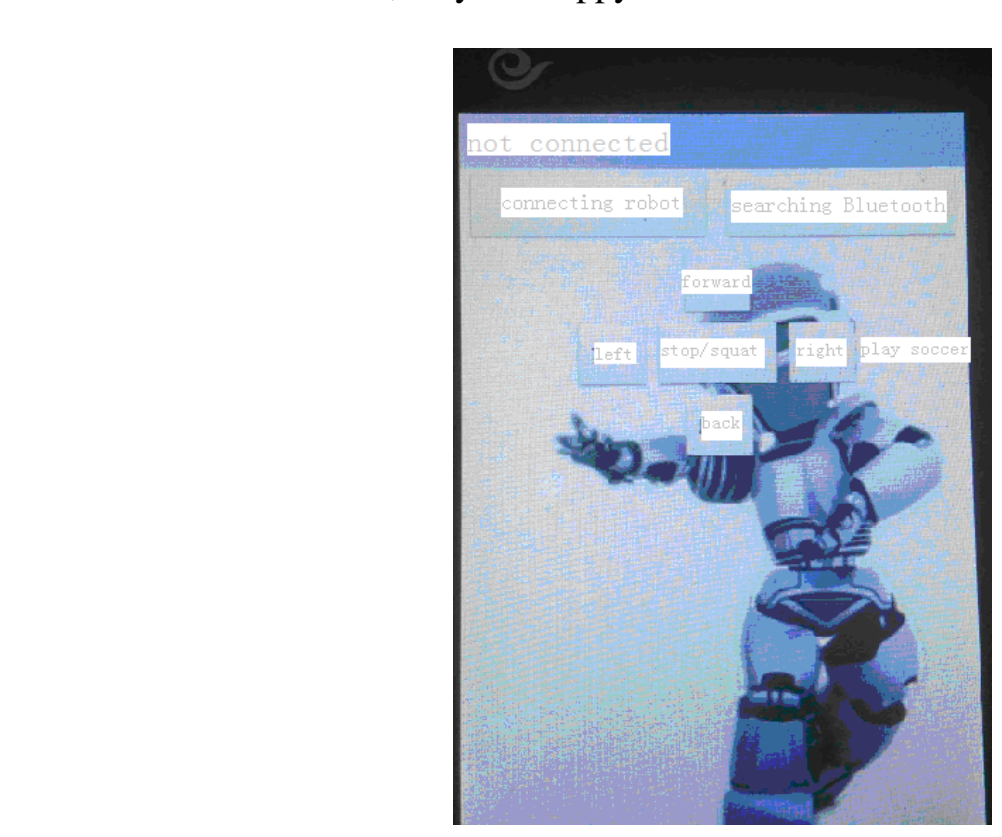

Figure 5. The Graphical Interface of the Android System 


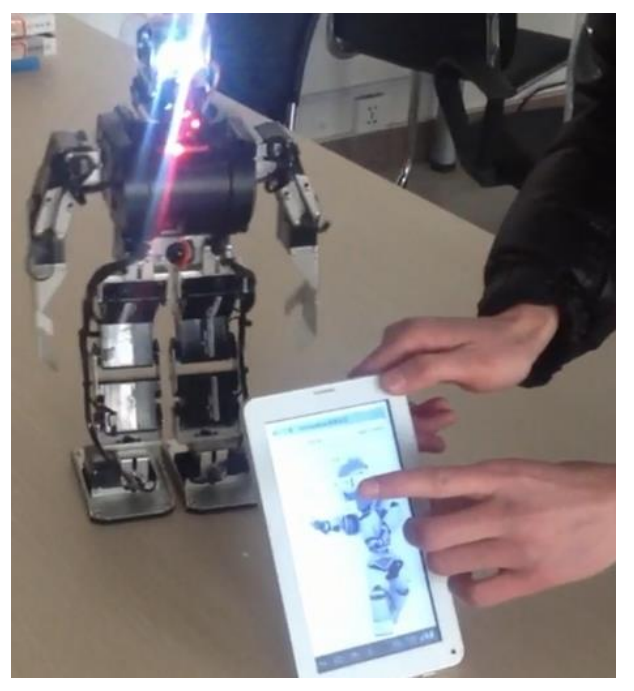

Figure 6. The Smart Phone Connected with the Robot

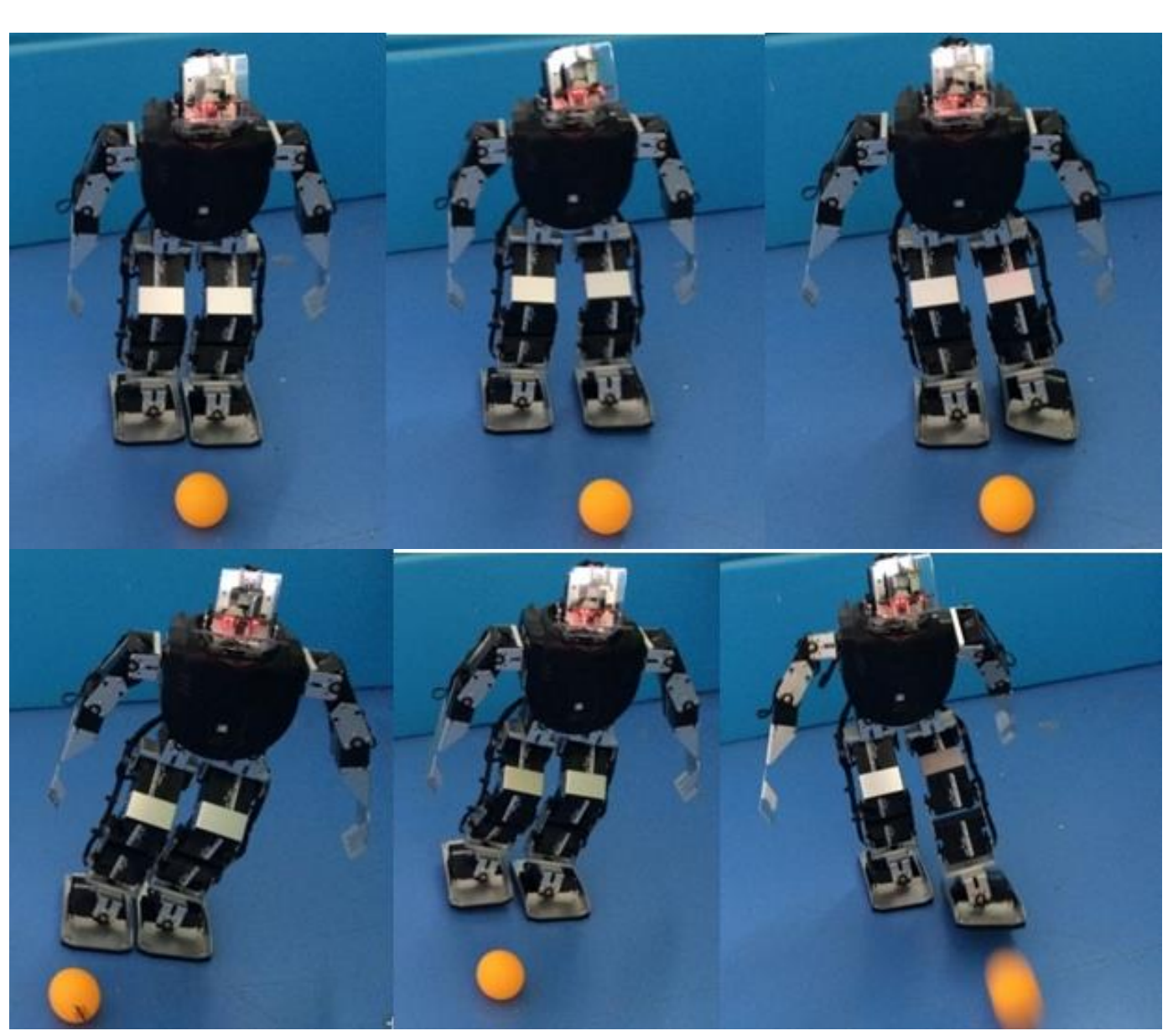

Figure 7. The Robot Playing Table Tennis with the Operator

Some core codes are shown as follows.

public class MainActivity extends Activity \{ 
private static final int FOUND_DEVICE $=1$;

private static final int START_DISCOVERY $=2$;

private static final int FINISH_DISCOVERY $=3$;

private static final int CONNECT_FAIL $=4$;

private static final int CONNECT_SUCCEED_P $=5$;

private static final int CONNECT_SUCCEED_N $=6$;

ConnectedThread connectedThread;

ConnectThread connectThread;

AcceptThread acceptThread;

private Button bLink, bShow,bt_go,bt_down,bt_lift,bt_right,bt_stand;

private TextView stateText;

private Dialog dialog;

private ProgressBar discoveryPro;

private ListView foundList;

List<BluetoothDevice> foundDevices;

BluetoothSocket socket;

BluetoothAdapter bluetoothAdapter = null;

private Handler $\mathrm{mHandler}=$ new Handler ()

@Override

public void handleMessage(Message msg) \{

// TODO Auto-generated method stub

switch (msg.what)

case FOUND_DEVICE: found $\overline{d i s t}$ setAdapter new

MyAdapter(MainActivity.this,foundDevices)),

case START_DISCOVERY:

2) discovelyr ro.setVisibility(View.VISIBLE);

break;

case FINISH DISCOVERY:

break;
case ConNECT_FAIL:
Toast.makeText(MainActivity.this, "failure",

Toast.LENGTH_SHPRT)

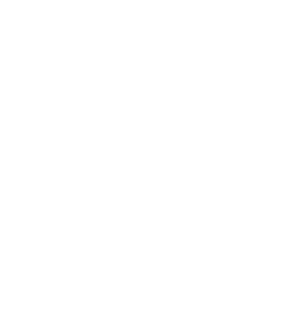

break;

.show ()

case CONNECT_SUCCEED_P:

case CONNECT_SUCCEED_N:

System.out.println("connecting success-----");

if (msg. what ==CONNECT_SUCCEED_P) \{

if (acceptThread != null) \{

\} acceptThread.interrupt();

socket $=$ connectThread.getSocket () ;

connectedThread $=$ new ConnectedThread $($ socket,

mHandler);

connectedThread.start();

\} else \{ 
mHandler);

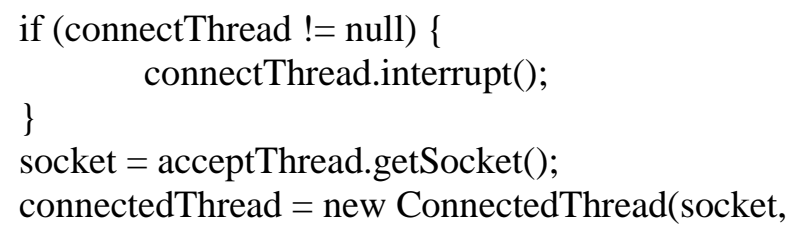

\section{Conclusion}

This project can be used to accompany the elderly from the unique perspective. In the smart telephone, they open the software, and control the robot to do all kinds of actions. They also play table tennis with the robot. They enjoy themselves. This project finished that the operator uses the intelligent phone to control the robot to move or dance. This will be applied widely in developing the smart home system.

\section{Acknowledgements}

This paper are sponsored by the Education Department of Anhui Province Key Natural Science Research Foundation of China under Grant (KJ2010A352), Suzhou University student scientific research initiating projects (KYLXLKYB14-35, KYLXLKYB 14-36, KYLXDKZD14-19), University science research project of Anhui Province (KJ2011B180, KJ2012A263, KJ2012B185), Quality project of Anhui Province (2012jyxm545) and Quality project of Suzhou University, The course construction of things of internet in the collaborative innovation environment (szxyjyxm201314), The IT class professional engineering practice teaching quality guarantee system construction (szxyzlbz201305IT). The innovation training program projects for the Anhui College students, Coal Mine Personnel Positioning System based on the RFID (201310379083), The implementation of embedded alarms management system based on A900 (20131037199023). Anhui Province College Humanities and Social Sciences Key Research Base - Culture University Research Center, the tender: Research on Campus Culture Construction Evaluation System (SK2014A116).

\section{References}

[1] X. Li and B.-J. Choi, "Design of Obstacle Avoidance System for Mobile Robot using Fuzzy Logic Systems", International Journal of Smart Home, vol. 7, no. 3, (2013), pp. 321-328.

[2] J. Jin-gang, Z. Yong-de, J. Ming-liang and W. chun-ge, "Bending Process Analysis and Structure Design of Orthodontic Archwire Bending Robot", International Journal of Smart Home, vol. 7, (2013), no. 5, pp. 345352. 
[3] Z. Xin and G. Chen, "The intelligent transportation system using the infrared sensors based on the ZigBee protocol and Eclipse", Sensors \& Transducers, vol. 158, (2013), pp, 94-99.

[4] X. Zhenghua, S. Qixiang and H. Liangyi, "Research on the Wireless Audio Transmission System based on the Infrared", Information Technology Journal, vol. 12, no. 23, (2013), pp. 7610-7615.

[5] K. Qian, X. Ma, C. Peng, Q. Ju and M. Xu, "A ZigBee-based Building Energy and Environment Monitoring System Integrated with Campus GIS”, International Journal of Smart Home, vol. 8, no. 2, (2014), pp. 107114.

[6] M. Mosleh, H. Noori and A. Nikdel, "Cluster Based and Energy Efficient Coverage Protocol for Wireless Sensor Networks", International Journal of Smart Home, vol. 8, no. 2, (2014), pp. 39-54.

[7] J. Song, Z. Xin and W. Ding, "Research on android intelligent phones controlling the car to run", TELKOMNIKA, vol. 11, no. 12, (2013), pp. 7438-7445.

[8] S. Kim, H. Kim, H. Ko and H. Yoe, "Design and Implementation of Android-based Livestock Disease Forecasting System using Thermal Image”, International Journal of Smart Home, vol. 7, no. 6, (2013), pp. 305-312.

[9] H. Lee and J. Kwon, "Ontology Model-based Situation and Socially-Aware Health Care Service in a Smårt Home Environment”, International Journal of Smart Home, vol. 7, no. 5, (2013), pp. 239-250.

[10] J.-V. Lee, Y.-D. Chuah and K. T. H. Chieng, "Smart Elderly Home Monitoring System with an Android Phone", International Journal of Smart Home, vol. 7, no. 3, (2013),pp. 17-32.

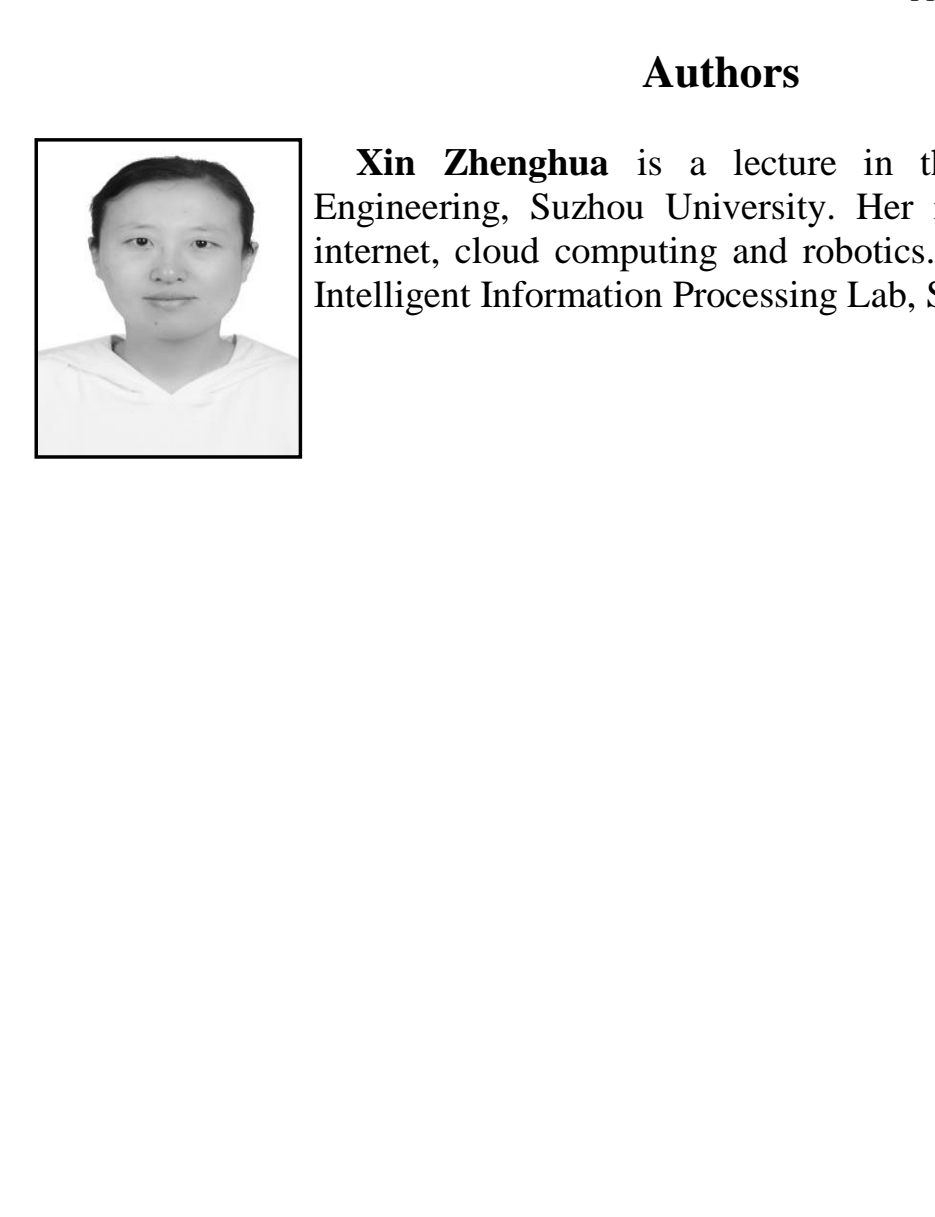

